\title{
Funciones (meta)pragmáticas de las interjecciones emotivas en edad temprana ${ }^{1}$
}

\section{(Meta)pragmatic functions of emotive interjections in early childhood}

\author{
GABRIELA PREGO VÁZQUEZ ${ }^{1}$, IVÁN ENRÍQUEZ MARTÍNEZ² \\ ${ }^{1}$ Facultad de Filología, Universidad de Santiago de Compostela, España \\ Correo electrónico: gabriela.prego@usc.es \\ ${ }^{2}$ Facultad de Ciencias de la Educación, Universidad de La Coruña, España \\ Correo electrónico: ivan.enriquez@udc.es
}

Este trabajo se centra en las interjecciones primarias emotivas en edad temprana. La investigación analiza una muestra del corpus de habla infantil Koiné. El estudio revela que las interjecciones, partículas consideradas en la periferia del sistema lingüístico, desempeñan un papel clave en el desarrollo pragmático puesto que sus usos tienden un puente entre la fase preidiomática y la idiomática. Los resultados de la investigación prueban la relevancia cuantitativa de estas partículas en edad temprana y, por su parte, el análisis pragmático muestra que las interjecciones constituyen actos proto-expresivos con función deíctica y que, ligadas a la indexicalidad, afloran sucesivamente nuevas funciones (meta)pragmáticas.

Palabras clave: interjecciones, deíxis, proto-expresivos, desarrollo pragmático, habla infantil.

The present paper deals with the study of primary emotive interjections in early childhood. We have analyzed data that belong to Koiné Child Language Corpus. The research shows that interjections particles which are considered as periphery within the linguistic system- play a key role in the pragmatic development of children since their uses link the preverbal with the verbal stage. Our results demonstrate the quantitative importance of these particles in early childhood. For its part, the pragmatic analysis has proved that, on the one hand, interjections constitute proto-expressive speech acts with deictic function and, on the other, new metapragmatic functions appear successively connected to indexicality.

Key words: interjections, deixis, proto-expressives, pragmatic development, child language

\footnotetext{
1 Esta investigación se ha desarrollado en los proyectos Exploración de capacidades metalingüisticas na linguaxe infantil 10PXIB204091PR (Xunta de Galicia) y Superdiversidad lingüistica en áreas periurbanas. Análisis escalar de procesos sociolingüisticos y desarrollo de la conciencia metalingüistica en aulas multilingües FI201676425-P (Ministerio Economía y Competitividad). Asimismo, Iván Enríquez Martínez ha contado con la financiación del Ministerio de Educación, Cultura y Deporte (FPU: AP2010-4723).
} 


\section{INTRODUCCIÓN}

Este artículo explora el papel de las interjecciones primarias emotivas (Ameka 1992) en el desarrollo pragmático. Se pretende desentrañar su funcionamiento como recursos que los niños usan para expresar sus emociones en edad temprana ${ }^{2}$. La investigación analiza una muestra del corpus de habla infantil Koiné. Concretamente, se han seleccionado 46 transcripciones del corpus que incluyen producciones lingüísticas en castellano de 56 niños (28 niños y 28 niñas) del período temporal que va desde 1;07 a 4;05 años ${ }^{3}$. La combinación de las variables niño y edad ha permitido establecer 161 individuos. Finalmente, se han constituido grupos de edad con una frecuencia de 6 meses para abordar el análisis.

Metodológicamente, se combina el análisis cuantitativo y cualitativo. En primer lugar, se identifican las interjecciones primarias emotivas, esto es, aquellas que señalan sentimientos, emociones y actitudes de los niños hacia un hecho y cuyas fuerzas ilocucionarias, por lo tanto, se corresponden con los markings (=actos de señalar) del sistema de codificación propuesto para fuerzas ilocucionarias en CHAT (Codes for the Human Analysis of Transcripts) del sistema CHILDES (MacWhinney 2000). Se identificaron y codificaron cuatro tipos de fuerzas ilocucionarias de las descritas en CHAT: EM (expresar dolor o molestia), EN (expresar emoción positiva), ED (exclamar en desaprobación) y ES (expresar sorpresa). Posteriormente se cuantifica (a) su uso distribuido por edades y (b) los subtipos de intenciones comunicativas expresivas movilizadas por estas partículas. Finalmente, los usos de las interjecciones se someten a un análisis pragmático y sociointeraccional cualitativo.

El estudio revela que las interjecciones, partículas consideradas en la periferia del sistema lingüístico, tienen una especial relevancia cuantitativa y cualitativa en edad temprana. Las interjecciones o "no-palabras" (Ameka 1992: 105) funcionan como actos proto-expresivos, esto es, son marcadores deícticos cuya actuación se limita exclusivamente al plano local "contextually situated here and now" (Montes 1999: 1317). Constituyen la forma más primitiva de los actos de habla expresivos ${ }^{4}$ y desempeñan un papel clave en el desarrollo pragmático puesto que sus usos tienden un puente entre la fase preidiomática y la idiomática. El análisis cualitativo también muestra que, ligada a la función deíctica,

2 Con el término "edad temprana" se alude, generalmente, al desarrollo lingüístico de los niños durante el periodo preescolar, en él se adquieren los recursos gramaticales básicos de la lengua y que suele culminar, aproximadamente, a los 5;0-6;0 años. De aquí en adelante, por tanto, aludiremos a "usos tempranos" para referirnos a este periodo cronológico.

3 Tradicionalmente, en el campo de estudio de la adquisición del lenguaje existen unas convenciones para expresar la edad de los niños, y que también han sido adoptadas en el presente trabajo: "años;meses.días". Por ejemplo, en este caso 1;07 significa un año y siete meses, mientras que 4;05 se refiere a una edad de cuatro ańos y cinco meses.

4 De acuerdo con la teoría de los actos de habla, un acto expresivo hace referencia a cómo el hablante se siente con respecto a la situación comunicada, o a su actitud ante la misma, esto es, alude a un estado afectivo emocional o físico (Searle 1969: 38-39). 
afloran sucesivamente nuevas funciones (meta)pragmáticas en la franja de edad estudiada. En definitiva, este trabajo pretende aportar nuevos datos para entender el desarrollo comunicativo de los actos de habla en edad temprana, tema escasamente tratado (vid. Prego Vázquez et al. 2011).

\section{LOS ESTUDIOS SOBRE INTERJECCIONES: CONTROVERSIA EN TORNO A LAS "NO-PALABRAS" Y A SU ADQUISICIÓN}

Las interjecciones son partículas universales para expresar emociones que están presentes en todas las lenguas. Se consideran elementos situados en la periferia del sistema lingüístico e incluso algunos autores las tratan como elementos paralingüísticos vinculados con el gesto en la frontera del plano verbal y no-verbal (Ameka 1992: 112; Goffman 1981: 122; Wilkins 1992). Así, el hecho de que los niños movilicen en sus primeros enunciados interjecciones y la naturaleza paralingüística de las mismas las convierte en elementos clave para "comprender la transición del niño hacia el lenguaje" (Alonso-Cortés Fradejas 2006: 64), cuestión que se ha señalado en diferentes trabajos a los que aludiremos en este apartado.

Las interjecciones son morfológicamente invariables y sintácticamente independientes. Desde el punto de vista pragmático, constituyen por sí mismas enunciados que "express a speaker's mental state, action or attitude or reaction to a situation" (Ameka 1992: 106). No poseen significado proposicional puesto que no constituyen representaciones simbólicas del mundo. Sin embargo, indexicalizan diferentes sentimientos y actitudes (Searle 1969: 38-39). Como señala Vázquez Veiga (2003: 180), siguiendo la distinción semántica entre comunicar y señalar establecida por Iordanskaja y Mel’čuk (1995), constituyen unidades léxicas no descriptivas. En este sentido, las interjecciones funcionan como marcadores discursivos (Ameka 1992; Blas Arroyo 1995; Montes 1999; Schiffrin 1987; o Vázquez Veiga, 2003, entre otros).

Tradicionalmente se han distinguido dos tipos de interjecciones: las interjecciones primarias y las interjecciones secundarias. Las interjecciones primarias, objeto de este estudio, son gestos vocales convencionalizados como $a h$, ay, bah, puffo uy, entre otros, que se producen como reacción al contexto lingüístico y no-lingüístico inmediato (Ameka 1992: 106-108). En cambio, las interjecciones secundarias son "sintagmas originariamente pertenecientes a otras categorías que han experimentado un proceso de inmovilización morfológica y de pérdida de función referencial originaria" (Rodríguez Ramalle 2007: 113). Es el caso, por ejemplo, de lástima, mira o fijate, que sólo funcionarán como interjecciones cuando "they can occur by themselves non-elliptically as one-word utterances and in this usage refer to mental acts" (Ameka 1992: 105).

Desde una perspectiva pragmática, se proponen clasificaciones basadas en las funciones que pueden desempeñar las interjecciones en el discurso. En esta línea se enmarca la propuesta de Ameka (1992), que aborda los tipos de interjecciones a partir de las funciones del lenguaje propuestas por Bühler (1934) y Jakobson (1960). Distingue entre 
interjecciones expresivas, conativas y fáticas. Las interjecciones expresivas son aquellas que sirven para manifestar el estado mental de los hablantes. Pueden ser emotivas y cognitivas. Las emotivas expresan el estado de las emociones del hablante, mientras que las cognitivas son aquellas que marcan el estado de conocimiento de los hablantes en el tiempo de la emisión. Por su parte, las interjecciones conativas reclaman la atención del oyente y, por último, las interjecciones fáticas se usan para establecer y mantener el contacto en la cadena comunicativa. Concretamente, las interjecciones analizadas en este trabajo responden al tipo expresivo emotivo.

La peculiar naturaleza de las interjecciones ha despertado interés desde los pensadores de la antigüedad clásica hasta la actualidad. Su identificación como parte de la oración ha sido un tema de discusión de gramáticos griegos y latinos como Dionisio de Tracia y Donato (Vázquez Veiga 2003: 41; Ameka 1992), de los modistas en el medievo y de importantes lingüistas del siglo XX como Jespersen (1924) o Bloomfield (1933), entre otros. Incluso sociólogos como Goffman (1981) han reparado en la importancia comunicativa de estas partículas.

Sin embargo, la dificultad para definir las interjecciones y situarlas en la gramática ha provocado que no existan estudios detallados de su funcionamiento hasta finales del siglo XX y principios del siglo XXI en el marco de la Pragmática y del Análisis de la Conversación. En este nuevo marco epistemológico, que transciende la gramática, las interjecciones se incluyen en la categoría de los "marcadores discursivos" y comienzan a ser tratadas como un elemento clave de las interacciones cara a cara (Blas Arroyo 1995; Ehlich 1986; Schegloff 1982; Schiffrin 1987; Vázquez Veiga 2003). Como señalan Meng y Schrabback (1999: 1264):

The interjections have the general function of establishing a direct relation between speaker and listener in discourse. This direct relation enables the participants to guarantee an elementary interactional conformity with regard to contact as a whole, with regard to the partners' emotional state, with regard to their processing of the information gained by the conversation, and with regard to future communicative acts and turn-taking (Ehlich, 1986: 241). Interjections are not natural sounds, but verbal entities. That means that they have to be learned (1999: 1264).

Los estudios sobre las interjecciones en el habla infantil son realmente escasos a pesar de que su uso es especialmente relevante en edad temprana. De hecho, la importancia de las interjecciones en el proceso de adquisición del lenguaje es subrayada en los diarios de bebés, considerados los primeros trabajos científicos sobre adquisición del lenguaje (Alonso-Cortés Fradejas 2006). En el diario lingüístico que Preyer (1882) realizó de su hijo Axel destacó el papel de las interjecciones en el proceso de adquisición de lenguaje. Asimismo, los diarios de bebés registrados por Brandenburg (1915) y Nice (1917) observan la introducción de las interjecciones en el habla infantil. De hecho, Alonso-Cortés Fradejas, partiendo de estos trabajos pioneros del lenguaje infantil, considera que el estudio de los 
fenómenos paralingüísticos, entre los que incluye a las interjecciones, son fundamentales para "comprender la transición del niño hacia el lenguaje" (2006: 64).

Las primeras investigaciones que desarrollan en mayor o menor medida este tema aparecen tímidamente en trabajos de la década de los setenta y de los ochenta (Bloom 1973; Gopnik 1982; Tesnière 1976; o Yairi 1981). Debemos esperar hasta la década de los noventa para encontrarnos con diferentes autores que se centran ya en la adquisición de las interjecciones. Por ejemplo, la investigación de Plunkett y Strömqvist (1992) sobre adquisición de backchannels en danés y sueco incluye la adquisición de las interjecciones. Igualmente, cabe destacar el trabajo de Asano (1997), que se ocupa de tres interjecciones inglesas concretas -ouch, yuck y oops- y defiende que son partículas que forman parte de las lenguas, no son emisiones automáticas de la comunicación y, por lo tanto, se tienen que integrar en los análisis de adquisición lingüística. De todas formas, no será hasta la aparición del trabajo de Strange (2009), que retoma en muchos aspectos el de Asano (1997), cuando se disponga de un estudio relativamente amplio y verdaderamente centrado en el desarrollo infantil de las interjecciones en la lengua inglesa, un terreno en el que todavía queda mucho por hacer. Strange (2009) indaga en el uso infantil de siete interjecciones que codifican tres intenciones comunicativas diferentes y llega a la conclusión de que el input adulto desempeńa un importante papel en el proceso gradual de adquisición y desarrollo de estas partículas.

Como podemos comprobar, el uso temprano de las interjecciones no es, en absoluto, un tema que haya suscitado el interés de los autores, y nuestra tradición investigadora no es una excepción en este sentido. Hasta donde nuestro conocimiento alcanza, solamente existen dos trabajos que hayan tratado la cuestión: el estudio pionero de Barriga (1992), que se ocupa de varios elementos expresivos entre los que se encuentran las interjecciones, y el artículo de Montes (1999), que constituye el único estudio exclusivamente centrado en la adquisición y el desarrollo de este tipo de partículas en el habla de sujetos que tienen el español como primera lengua. La investigación llevada a cabo por Montes es especialmente interesante puesto que trata las interjecciones en edad temprana como marcadores de cambio de estado de conocimiento que proyectan o apuntan hacia un referente externo (objeto, evento en el contexto o emisión discursiva). Su trabajo muestra que el proceso de adquisición de estas partículas revela que hay una progresión de los usos contextuales hacia los usos discursivos y metafóricos (Montes 1999: 1319).

\section{Datos y Metodología}

La muestra de datos manejada para la realización del presente trabajo procede del corpus de habla infantil Koiné. Esta base datos, que se integra en el sistema CHILDES (Child Language Data Exchange System) (Snow y MacWhinney, 1985), contiene producciones espontáneas de 64 sujetos (30 niños y 34 nińas) con edades comprendidas entre los 1;07 $\mathrm{y}$ los 4;05 ańos y que han recibido un seguimiento longitudinal que oscila entre los seis 
y los veinticuatro meses. Para su confección se han seguido los pasos establecidos por la metodología RETAMHE: Registro, Transcripción y Análisis de Muestras de Habla Espontánea (Díez-Itza, Snow y MacWhinney 1999).

Así pues, hemos recurrido a la observación natural del comportamiento comunicativo de los niños, lo que nos ha permitido disponer de datos de habla infantil a partir de muestras orales, espontáneas y adecuadamente contextualizadas. Precisamente con el objetivo de que su comportamiento fuese lo más espontáneo y natural posible, los sujetos del corpus Koiné eran grabados audiovisualmente en sus propias guarderías. Cada quince días, y durante un periodo que se extiende hasta los cuatro años (entre 1996 y 2000), un investigador acudía a las escuelas infantiles y, asumiendo en ocasiones un papel más activo y en otras un papel más pasivo, mantenía conversaciones con los niños, habitualmente cuatro o cinco, en diferentes contextos interaccionales: juegos, pruebas de denominación, lectura de cuentos, relatos de acontecimientos, etc. La selección de las guarderías se realizó tratando de respetar la variedad lingüístico-cultural de Galicia, por lo que se escogieron cinco (Breogán, Elfos, Milagrosa, Santa Susana y Vite) ubicadas en tres poblaciones diferentes (A Estrada, Lugo y Santiago de Compostela) y en zonas, tanto urbanas como rurales, cuya población responde a diferentes niveles socioculturales.

A continuación, los datos recogidos se transcribían de acuerdo con las convenciones establecidas por el formato CHAT (Codesfor the Human Analysis of Transcripts) (MacWhinney 2000), que comparten todos los corpus que constituyen la base de datos internacional CHILDES. Este formato se caracteriza por su rigor $\mathrm{y}$, a la vez, por su sencillez y flexibilidad, por lo que permite realizar ajustes y modificaciones en función de los intereses particulares de cada investigación, opción de la que, como se verá en el apartado 3.2., hemos decidido aprovecharnos. Además, cuenta con la ventaja de llevar asociado un paquete de programas informáticos, CLAN (Computerized Language Analysis), que agiliza enormemente el paso final, el análisis (\$3.3.).

\subsection{Muestra seleccionada para el estudio}

El corpus Koiné está integrado por un total de 166 transcripciones de las que, para el presente estudio, hemos seleccionado una muestra de 46. Esta selección responde fundamentalmente a dos intereses principales. Por un lado, nuestro propósito era contar con datos procedentes de las cinco escuelas infantiles de las que consta el corpus y con una cadencia temporal de tres meses, siempre que las grabaciones lo permitiesen. Presentamos en el Cuadro 1 las transcripciones que hemos manejado: 
Cuadro 1. Listado de transcripciones seleccionadas

\begin{tabular}{|c|c|c|c|c|c|}
\hline $\begin{array}{l}\text { Escuela } \\
\text { infantil }\end{array}$ & $\begin{array}{l}\text { Número de } \\
\text { transcripciones }\end{array}$ & $\begin{array}{c}\text { Fecha de la } \\
\text { primera grabación }\end{array}$ & $\begin{array}{l}\text { Fecha de la } \\
\text { última grabación }\end{array}$ & $\begin{array}{l}\text { Duración } \\
\text { total }\end{array}$ & $\begin{array}{l}\text { No total de } \\
\text { individuos }\end{array}$ \\
\hline $\begin{array}{c}\text { Breogán } \\
\text { (Santiago) }\end{array}$ & 12 & 06-nov-96 & 15 -jun-00 & 3h. 20' 14" & 55 \\
\hline $\begin{array}{c}\text { Elfos } \\
\text { (A Estrada) }\end{array}$ & 11 & 08-nov-96 & 22-dic-98 & 2h. 45' 7" & 30 \\
\hline $\begin{array}{c}\text { A Milagrosa } \\
\text { (Lugo) }\end{array}$ & 5 & $10-f e b-99$ & 24-may-00 & 1h. 22' $58^{\prime \prime}$ & 14 \\
\hline $\begin{array}{c}\text { Santa Susana } \\
\text { (Santiago) }\end{array}$ & 7 & 21-may-97 & 27-may-99 & 2 h. $5^{\prime} 25^{\prime \prime}$ & 29 \\
\hline $\begin{array}{c}\text { Vite } \\
\text { (Santiago) }\end{array}$ & 11 & 05-nov-97 & 08 -jun- 00 & 2h. 34' 24" & 33 \\
\hline
\end{tabular}

Por otra parte, esta selección recoge las producciones de la mayoría de los niños del corpus manejado -56 nińos de un total de 64- y de todo el período temporal recogido en el mismo (1;07-4;05). A través de la combinación de las variables niño y edad se han establecido 161 individuos, puesto que, en general, los participantes del corpus participan en varias sesiones de grabación a lo largo de su evolución temporal. La selección de los sujetos se realizó de acuerdo con varios parámetros: el equilibrio en el género (28 niños y 28 niñas), la variada extracción social y el uso exclusivo o mayoritario del castellano frente al gallego. Puede consultarse la lista completa de individuos en Prego et al. (2011: 160-161).

\subsection{El proceso de codificación pragmática del corpus Koiné}

El corpus Koiné, en su versión actual -disponible en la dirección web http://childes. psy.cmu.edu/data/Romance/Spanish/koine-, carece de una codificación pragmática ${ }^{5}$. Por ello, una vez seleccionadas las transcripciones y los sujetos, hemos decidido aprovecharnos de la

\footnotetext{
$5 \quad$ Hasta el momento, en el corpus Koiné se han codificado usos peculiares del lenguaje infantil, señalando los componentes en que se inscriben (fonología, morfología y sintaxis), así como propiedades morfosintácticas de las construcciones verbales (cf. Fernández Pérez 2011: 26.36). Sin embargo, dado que el proceso de etiquetado es paulatino y es una labor que todavía no ha terminado, el corpus no dispone todavía de una codificación pragmática, esto es, relativa a las funciones de las unidades en el discurso y a las acciones e intenciones comunicativas de los nińos. Este trabajo, por tanto, pretende avanzar en esta dirección, con objeto de anotar características relevantes del desarrollo pragmático, habida cuenta que este componente motiva y conduce el desarrollo gramatical en las edades tempranas (Prego Vázquez et al. 2011). Disponer de esta información, pues, resulta fundamental para conocer cómo se desarrollan el resto de componentes.
} 
codificación de los actos de habla realizada en Prego et al. (2011), que han recurrido al sistema propuesto en el manual CHAT del sistema CHILDES (MacWhinney 2000). Este sistema parte del inventario de actos comunicativos presentados por Ninio et al. (1994), el INCA-A (Inventory of Communicative Acts - Abridged), que simplifica una clasificación previa de Ninio y Wheeler (1986). Ambas taxonomías fueron especialmente diseñadas para el estudio del lenguaje infantil en las edades tempranas, concretamente las reglas de producción empleadas por los niños para verbalizar intenciones comunicativas en las interacciones en que participan.

Teniendo en cuenta los objetivos del presente estudio, nuestro interés residía exclusivamente en los actos de habla expresivos, en cuyo interior se distinguen cinco tipos de intenciones comunicativas diferentes, como se observa en el Cuadro 2:

Cuadro 2: Listado de actos de habla expresivos

\begin{tabular}{ll}
\hline & ED: exclamar en desaprobación \\
& EM: expresar dolor o molestia (física o psíquica) \\
Actos de habla expresivos & EN: expresar emoción positiva \\
& ES: expresar sorpresa \\
& MK: saludar, agradecer, disculparse, etc. \\
\hline
\end{tabular}

Con estas cinco etiquetas se procedió a codificar todas las interjecciones expresivas halladas en las transcripciones y en los sujetos seleccionados, aunque una de ellas, MK, nunca se manifiesta en el corpus Koiné mediante este tipo de partículas y, por consiguiente, no fue necesario recurrir a su utilización en ningún momento. Para localizar las interjecciones, y proceder a su codificación pragmática, fue necesario etiquetarlas previamente en las líneas principales de la transcripción mediante el código "@i”, que es el que se recomienda en el manual del formato CHAT para tal efecto (MacWhinney 2000: $\$ 7.3$.). Es decir, esta etiqueta se añade al final de la interjección que corresponda - por ejemplo, "jo@i”- en aquellas líneas que recogen las producciones verbales de los participantes.

\subsection{Metodología de análisis}

El tercer y último paso de la metodología RETAMHE, como hemos dicho, es el análisis, que se agiliza enormemente gracias a la existencia del paquete de programas informáticos CLAN, asociado con el formato de codificación CHAT. En concreto, se manejaron dos herramientas: FREQ, con la que se localizaron todas las interjecciones movilizadas por los participantes, y KWAL, con el que se extrajo un fragmento contextual suficientemente amplio como para poder valorar adecuadamente las intenciones comunicativas de los niños. 
Con estas herramientas informáticas pudimos realizar un análisis en el que se combina la metodología cuantitativa y cualitativa. Así, una vez identificadas y codificadas las cuatro fuerzas ilocucionarias descritas en CHAT -EM (expresar dolor o molestia), EN (expresar emoción positiva), ED (exclamar en desaprobación) y ES (expresar sorpresa)-, procedimos a cuantificar el número total de interjecciones expresivas registradas, el número de actos de habla expresivos y la distribución de las interjecciones expresivas por subtipos en el periodo de edad analizado. Además, y para mayor claridad en la presentación de los resultados, se constituyeron grupos de edad con una frecuencia de seis meses, por lo que también calculamos el porcentaje de subtipos de interjecciones expresivas por franjas de edad. Presentamos esta distribución en el cuadro 3:

Cuadro 3. Número de inviduos por grupo de edad

\begin{tabular}{|c|c|c|c|}
\hline Grupos de edad & Individuos & Grupos de edad & Individuos \\
\hline De $1 ; 07$ a $1 ; 11$ & 8 & De $3 ; 00$ a $3 ; 05$ & 51 \\
De $2 ; 00$ a $2 ; 05$ & 24 & De $3 ; 06$ a $3 ; 11$ & 26 \\
De $2 ; 06$ a $2 ; 11$ & 34 & De $4 ; 00$ a $4 ; 05$ & 9 \\
\hline
\end{tabular}

A continuación, las diferentes interjecciones expresivas, y sus subtipos, se someten a un análisis cualitativo de tipo pragmático y sociointeraccional con el fin de conocer los usos y funciones con las que los niños movilizan estas partículas. Hemos partido, para ello, de un marco multi-metodológico en el que se combina la Teoría de los actos de habla y los recursos del Análisis del discurso para analizar los marcadores. De este modo, pudimos distinguir varios tipos de usos y funciones de las interjecciones expresivas en el habla infantil que se agruparon, como se verá posteriormente (\$4.2.), en tres categorías fundamentales. Por último, también el análisis cualitativo nos ha permitido detectar ciertos usos metapragmáticos de las partículas analizadas que, aunque escasos, tienen una gran importancia en cuanto que precursores de funciones más avanzadas, propias del desarrollo posterior $(\$ 5)$.

\footnotetext{
$\overline{6}$ La teoría de los actos de habla es relevante en este estudio porque es clave para definir el objeto de estudio de esta investigación, esto es, aquellas interjecciones que movilizan intenciones comunicativas expresivas (ver también nota 3 ).
} 


\section{ANÁLISIS: USOS Y FUNCIONES DE LAS INTERJECCIONES EN EL CORPUS KOINÉ}

Las interjecciones son los primeros recursos que los niños tienen para señalar intenciones comunicativas expresivas en el puente entre lo preidiomático y lo idiomático El repertorio de interjecciones del corpus Koiné incluye 26 formas, a saber: $a h$, aah, ala, $a u$, ay, bah, boh, buu, eh, eeh, eiley, elo, hmm, iá, ja, jo, mmn, nn, oh, oiloy, ooh, puf, sss, uah, um, uy. En los siguientes apartados presentaremos los resultados del análisis cuantitativo $(\$ 4.1)$ y del análisis cualitativo $(\$ 4.2)$.

\subsection{Análisis cuantitativo}

El análisis de los actos de habla del corpus Koiné realizado en Prego et al. (2011: 169-170) muestra no sólo que los actos expresivos son el tipo que presenta las frecuencias y las medias más bajas con respecto al resto de tipos de actos de habla, sino que también se observa un predominio de interjecciones que funcionan como actos protoexpresivos. El siguiente cuadro ilustra esta distribución:

Cuadro 4. Distribución cuantitativa: interjecciones totales vs. actos de habla en el corpus

\begin{tabular}{|cccc}
\hline Grupos de edad & $\begin{array}{c}\text { Expresivos } \\
\text { movilizados por } \\
\text { interjecciones }\end{array}$ & $\begin{array}{c}\text { Actos de habla } \\
\text { expresivos totales }\end{array}$ & $\begin{array}{c}\text { Porcentajes de } \\
\text { interjecciones / actos } \\
\text { expresivos }\end{array}$ \\
\hline $\mathbf{1 ; 0 7}-\mathbf{1 ; 1 1}$ & 11 & 23 & $47,83 \%$ \\
$\mathbf{2 ; 0 0}-\mathbf{2 ; 0 5}$ & 6 & 10 & $60 \%$ \\
$\mathbf{2 ; 0 6}-\mathbf{2 ; 1 1}$ & 31 & 36 & $86,11 \%$ \\
$\mathbf{3 ; 0 0}-\mathbf{3 ; 0 5}$ & 28 & 50 & $56 \%$ \\
$\mathbf{3 ; 0 6}-\mathbf{3 ; 1 1}$ & 19 & 28 & $67,86 \%$ \\
$\mathbf{4 ; 0 0}-\mathbf{4 ; 0 5}$ & 11 & 18 & $61,11 \%$ \\
\hline Total & 106 & 165 & $64,24 \%$ \\
\hline
\end{tabular}

Las interjecciones primarias que funcionan como actos protoexpresivos en nuestra muestra de datos se idenfican con los markings (=actos de señalar) del sistema de codificación propuesto en CHAT. Concretamente, el 37,74\% corresponde a la expresión de sorpresa (ES), el 35,85\% sirve para señalar desaprobación (ED), el 21,70\% corresponde a la expresión de dolor o molestia (EM) y el 4,72\% son actos para expresar emoción positiva $(\mathrm{EN})$ : 


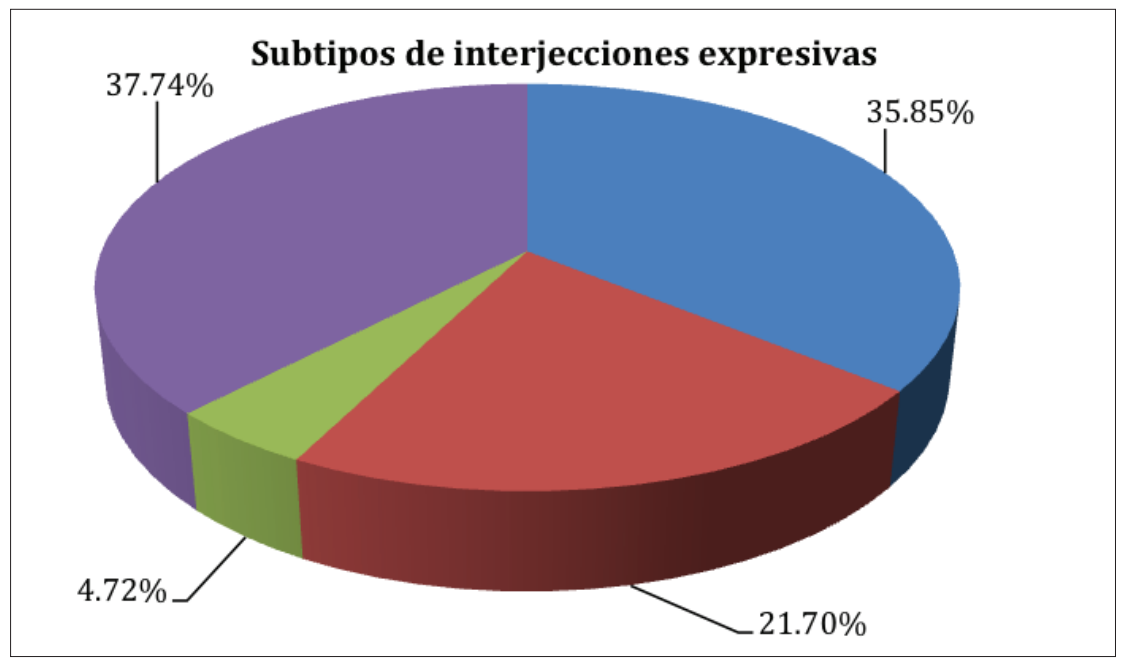

Figura 1. Subtipos de interjecciones expresivas

Estos cuatro subtipos presentan los siguientes porcentajes distribuidos por edades:

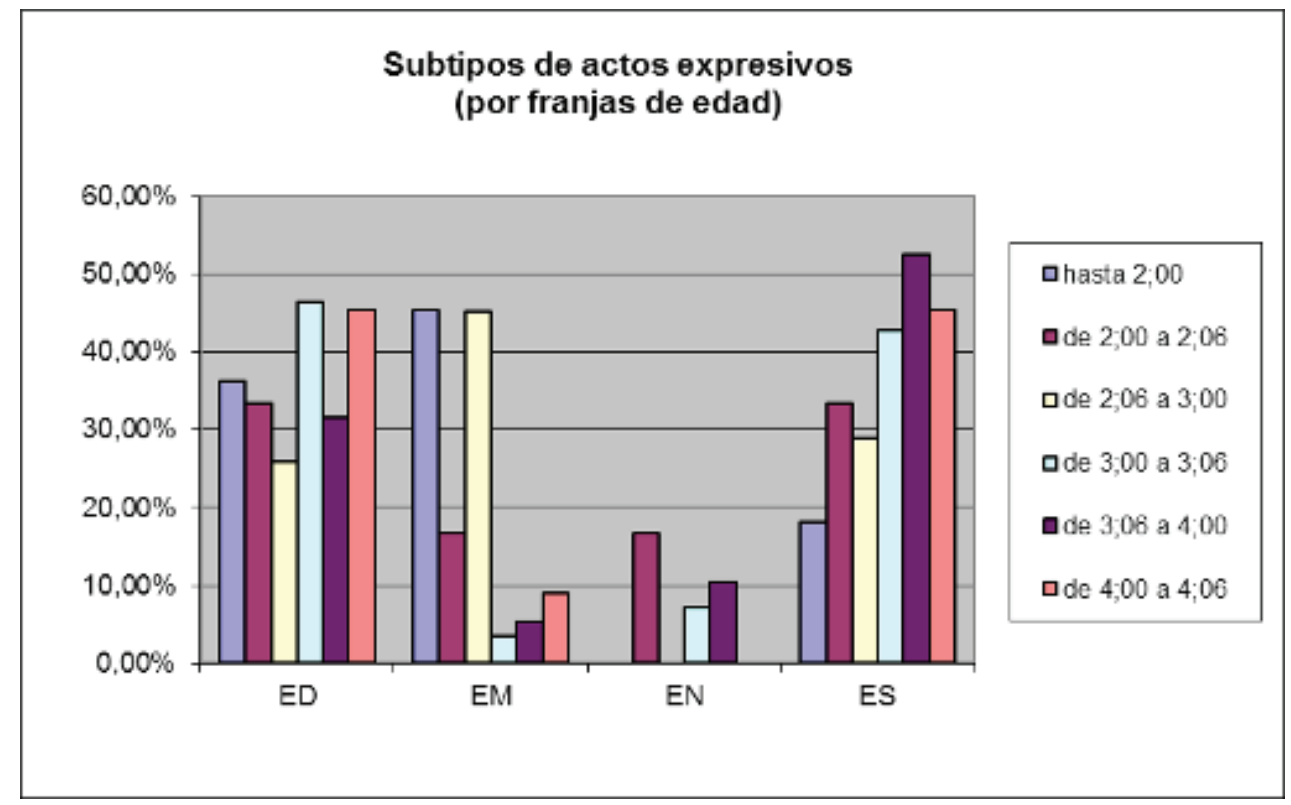

Figura 2. Porcentaje de subtipos interjecciones expresivas por edades. 
Este recuento muestra que el predominio del uso de las interjecciones se aprecia antes de los 2;0 años, lo que es una prueba de la función clave que estas partículas desempeñan en el tránsito entre la fase preidiómática a la idiomática.

\subsection{Análisis cualitativo}

La investigación cualitativa demuestra que las interjecciones funcionan en edad temprana como proto-expresivos con función deíctica (Wilkins 1992). Constituyen actos señalativos (Iordanskaja y Mel'čuk 1995) que actúan como marcadores proyectivos que operan en el ámbito local "contextually situated here and now" (Montes 1999: 1317). Asimismo, el análisis muestra que, ligadas a la función deíctica, afloran otras funciones pragmáticas. Así, basándonos en los resultados obtenidos, podemos establecer tres tipos de funciones, a saber: proyectiva contextual $(\$ 4.2 .1)$, proyectiva proto-textual $(\$ 4.2 .2)$ y proyectiva proto-modalizadora (\$4.2.3).

\subsubsection{Las interjecciones como marcadores proyectivos contextuales}

En este primer tipo, las interjecciones son elementos únicos del turno. Se movilizan para seńalar actitudes o sentimientos ante un hecho que ocurre en el contexto inmediato compartido por los participantes, y que no se representa verbalmente en la interacción. Por lo tanto, desempeñan una función deíctica contextual.

Las interjecciones como marcadores proyectivos contextuales constituyen la forma más primitiva de los actos expresivos. En nuestros datos las interjecciones actúan como protoexpresivos, enclavados en la frontera entre lo preidiomático y lo idiomático. Su significado deíctico y su uso contextual local constituirán la base de la primera fase del desarrollo de las intenciones comunicativas en edad temprana: la fase deíctica (Prego et al. 2011). De hecho, en nuestro corpus las primeras interjecciones con esta función se registraron a la edad de 1;10.18. Las interjecciones como marcadores proyectivos contextuales se usan para expresar cuatro de los cinco tipos de intenciones comunicativas que se detectan en los actos expresivos del corpus Koiné analizados en Prego et al. (2011). Concretamente, los subtipos detectados y codificados son: desaprobación (ED), expresar dolor o molestia física o psíquica (EM), expresar emoción positiva (EN) y expresar sorpresa (ES). Por ejemplo:

Ejemplo 1. Participantes: ART (niño), RIC (niño) y MON (adulta). Edad: RIC, 1;10.18. Grabación: elf1_01

\%exp: ART y RIC están jugando por separado, a los dos se les cae la construcción de juguete

*MON: cómo se llama esto ?

\%add: RIC

\%act: MON señala la construcción de RIC

*RIC: jo@i ! 
\%spa: \$i:ed ;

${ }^{*} \mathrm{MON}$ : jo (.) qué es (.) ah (.) se cayó .

\%com: no entendía si lo estaba nombrando o se quejaba por haberse caído la construcción

En este caso, Ricardo utiliza la interjección jo para expresar su molestia por un evento que acaba de producirse: la construcción de juguete se ha caído. Obsérvese que el turno de Ricardo sólo contiene la interjección para señalar su actitud ante el hecho ocurrido. Esta sería la forma más primitiva de un proto-acto de habla (proto-expresivo en este caso): la movilización de la interjección para señalar actitudes o sentimientos ante un hecho que sucede en el contexto inmediato, y que no se representa verbalmente en la interacción. Exactamente igual sucede en el siguiente ejemplo:

Ejemplo 2. Participantes: JUA (niño) y PIL (adulta). Edad:JUA, 2;07.08. Grabación: bre5_07

*PIL: una nada más .

*PIL: esta?

*PIL: a ver (.) enséñale a Xaquín (.) a ver que é ?

*JUA: oh@i !

\%spa: \$i:es ;

*PIL: qué?

*PIL: enséñalle a Xaquín a ver si sabe .

*JUA: <una paqui [?]> [//] (.) una ovejita .

\subsubsection{Las interjecciones como marcadores proyectivos proto-textuales ${ }^{7}$}

En nuestros datos las interjecciones también funcionan como marcadores deícticos de emisiones producidas en el mismo turno y que reproducen verbalmente un suceso del contexto inmediato. No se corresponden exactamente con los marcadores proyectivos textuales que analiza Montes (1999), puesto que los ejemplos analizados por esta autora responden a usos endofóricos y referentes textuales. Sin embargo, los proyectivos prototextuales constituyen un precedente de los usos textuales señalados por Montes (1999) y un avance con respecto a los usos puramente contextuales observados en el apartado 4.2.1.

\footnotetext{
Entendemos por marcadores proto-textuales un tipo de marcador de discurso propio de las primeras etapas del habla infantil. El análisis de los datos nos muestran que en edad temprana, además de la función de las interjecciones como marcador deíctico y contextual analizada en el 4.2.1, observamos otro tipo de función que todavía no se corresponde con usos claramente textuales (marcador endofórico) pero constituye un estadio intermedio entre esos usos y los meramente contextuales. Los ejemplos analizados en el 4.2.2. son relevantes para entender esta fase que ilustra el continuum entre lo contextual y textual.
} 
La combinación en el mismo turno de una interjección y de un enunciado es muy recurrente en los datos y presenta diferentes estructuras: (a) interjección + asertivo o asertivo + interjección, (b) interjección + expresivo iniciado por "qué" y (c) interjección + directivo.

\section{a) Interjección + asertivo}

Los resultados de este análisis muestran que en este tipo de estructura las interjecciones sólo se usan para expresar tres de las cinco intenciones comunicativas detectadas en los actos expresivos del corpus Koiné (Prego et al. 2011): desaprobación (ED), expresar dolor o molestia física o psíquica (EM) y expresar sorpresa (ES). Constituyen marcadores que proyectan la desaprobación, el dolor o la molestia física o psíquica al evento que se está reproduciendo lingüísticamente y que se está desarrollando en el contexto físico inmediato. Entre la interjección y el asertivo se establece meramente una relación deíctica. El siguiente ejemplo ilustra este tipo de función:

Ejemplo 3. Participantes: ART (niño), RIC (niño) y MON (adulta). Edad: RIC, 1;10.18. Grabación: elf1_01

${ }^{*} \mathrm{MON}$ : qué se te cayó (..) eso ?

\%add: RIC

\%act: RIC señala su construcción

${ }^{*} \mathrm{MON}$ : oh (.) se volvió a caer (.) qué mal .

${ }^{*} \mathrm{MON}$ : qué buscas (.) Ana?

*RIC: oh@i (.) cayó [*] !

\%xpar: cayó=se cayó \$CON \$MIX ;

\%spa: \$i:em ; \$i:st ;

*RIC: oh@i (.) cayó [*] !

\%xpar: cayó=se cayó \$CON \$MIX ;

\%spa: \$i:em ; \$i:st ;

*RIC: oh@i (.) cayó [*] !

\%xpar: cayó=se cayó \$CON \$MIX ;

\%spa: \$i:em ; \$i:st ;

\%com: juega a tirar lo que construye

En este caso, Ricardo repite hasta en tres ocasiones el mismo enunciado: oh@i (.) cayó $[*]$. La interjección oh constituye un proto-expresivo que señala molestia hacia el hecho enunciado y ocurrido en el contexto inmediato: las construcciones de juguete que se le han caído a él y a ART.

Cabe destacar que en nuestros datos hemos detectado una alteración en el orden de esta estructura, esto es, asertivo + interjección. Aunque no es un uso recurrente, consideramos que su aparición es relevante puesto que supone un avance con respecto a la función proto-textual que presenta la estructura interjección + asertivo. Así, mientras que en esta última estructura la interjección indexicaliza un cambio del estado de conocimiento, en 
los ejemplos de asertivo + interjección la partícula contribuye al manejo del flujo informativo del discurso, dado que proyecta su fuerza ilocucionaria sobre tópicos relanzados que ya han sido introducidos en el discurso previo. Por ejemplo:

Ejemplo 4. Participantes: JOR (niño), XUN (niño) y CHE (adulta). Edad: XUN, 2;11.15. Grabación: vit3b_07

*XUN: uy@i me cae el agua uy@i .

$\%$ spa: \$i:em ; \$i:st ;

${ }^{*}$ CHE: mira qué animal más feo .

*JOR: esa araña es mala .

\%spa: \$i:st ;

*XUN: esa araña es fea ah@i !

$\%$ spa: \$i:st; \$i:em;

${ }^{*}$ CHE: es fea y es mala .

*JOR: sí es mala .

\%spa: \$i:ap ;

b) Interjección + qué + expresivo

Se han detectado pocos ejemplos que respondan a este tipo y el primero se registra a los 2;11.15 ańos. Las interjecciones en estos casos proyectan emoción positiva (EN) o negativa (EM) a un enunciado exclamativo que apunta hacia un hecho o referente del contexto inmediato. Así se observa en los dos siguientes ejemplos:

Ejemplo 5. Participantes: JOR (niño), XUN (niño) y CHE (adulta). Edad: XUN, 2;11.15. Grabación: vit3b_07

*XUN: este es .

$\%$ spa: \$i:ap ;

*CHE: mira este aquí +//.

*CHE: ay qué animal más feo !

*XUN: uy@i qué culebra tan mala [!] .

\%spa: \$i:em ; \$i:st ;

${ }^{*}$ CHE: eso es una culebra a que sí ?

*JOR: es buena .

\%spa: \$i:dw ;

Ejemplo 6. Participantes: CES (niña), MIA (niña) y MON (adulta). Edad: MIA, 3;3.24. Grabación: mil1_07.

*MON: son caramelos?

*MIA: no .

\%spa: \$i:an ; 
*MIA: son de chocolate .

$\%$ spa: \$i:sa ;

*MIA: uy@i (.) qué ricas !

\%spa: \$i:en ; \$ist:

${ }^{*}$ CES: mía $\left.{ }^{*}\right]\left(\right.$.) oto $\left[{ }^{*}\right]$.

\%spa: \$i:jf; \$i:st ;

\section{c) Interjección + directivo}

Este tipo de construcción se produce normalmente cuando hay un cambio de percepción física asociado al ver o al oír. La interjección funciona como una marcador prototextual que proyecta su fuerza ilocucionaria al directivo y al hecho contextual señalado por éste. Como en el caso anterior, hemos detectado pocos ejemplos. Sin embargo, su análisis es pertinente porque ilustra anticipos de funciones textuales más elaboradas. La primera aparición en los datos corresponde a la edad de 2;09.27. El siguiente caso muestra esta función:

Ejemplo 7. Participantes: INE (niña) y AUR (adulta). Edad: INE, 2;09.27. Grabación: vit1_01.

*INE: yo pezuña [?] $\sin \mathrm{xxx}$.

\%spa: \$i:st ;

*INE: mira (.) mira!

$\%$ spa: \$i:jf;

\%com: otro niño le intenta quitar la plastilina

\%add: AUR

*INE: ay@i mira!

$\%$ spa: \$i:ed ; \$i:jf;

\%com: de nuevo intentan quitarle la plastilina

\%add: AUR

Observamos que Irene se dirige a la profesora (AUR) para solicitarle que mire cómo otro niño le intenta quitar la plastilina. Como la profesora no responde a esta demanda, Irene relanza el directivo encabezando esta vez el turno por la interjección ay para expresar desaprobación por la situación. Obsérvese que la interjección como marcador proto-textual proyecta esta fuerza ilocucionaria al directivo que le sigue y sirve también para conectarlo con el discurso precedente. Así pues, en este ejemplo la interjección como marcador prototextual tiene una función cohesiva que transciende el turno.

\subsubsection{Las interjecciones como marcadores proyectivos proto-modalizadores}

La construcción en la que se produce esta función es interjección + que + asertivo. Los resultados de este análisis muestran que en este tipo de construcción las interjecciones sólo 
se usan para expresar dos intenciones comunicativas: desaprobación (ED) y malestar (EM). La primera vez que aparece esta construcción en nuestros datos es a la edad de 3;00.16. La interjección aparece unida al asertivo a través de que, partícula que funciona en estos ejemplos como un marcador discursivo de proto-modalidad a través de cual el niño no sólo enfatiza y refuerza la aserción sino que también la vincula a la desaprobación emitida por la interjección (Diessel y Tomasello 2001; Givón 2009). De esta manera, la construcción interjección + que + asertivo se presenta, por un lado, como réplica de lo ocurrido en el contexto espacial inmediato y, por otro lado, enfatiza la expresión de desaprobación emitida en la interjección ${ }^{8}$. Así, la relación que se establece entre la interjección y el asertivo no es meramente deíctica como sucede en la construcción analizada en el apartado (a) de la sección 4.2.2. El conector que funciona como marcador de proto-modalidad porque sirve para permear la fuerza ilocucionaria de la interjección al asertivo. De este modo, interjección + que constituye un marcador de protomodalidad.

Ejemplo 8. Participantes: JOR (niño), XUN (niño) y CHE (adulta). Edad: XUN, 2;11.15. Grabación: vit3b_07.

*JOR: algunas arañas son buenas y unas [*] son malas .

\%spa: \$i:st ;

*CHE: y esto ?

*JOR: un pez bueno .

$\%$ spa: \$i:sa ;

*XUN: un pez bueno (.) ay@i que ma caigo .

\%spa: \$i:rt; \$i:em; \$i:sp ;

*JOR: $\mathrm{xxx}$.

\%spa: \$i:oo ;

${ }^{*}$ CHE: dónde vive $+/$.

Vemos que XUN recurre a esta construcción, y cabe destacar la diferencia con respecto al ejemplo 2 ( ${ }^{*}$ RIC: oh@i (.) cayó [*]) en el que se establece una relación meramente proyectiva y deíctica entre la interjección y el asertivo. En cambio, en el caso que ahora nos ocupa, la presencia de que como marcador discursivo establece, además de una conexión deíctica, una marca de proto-modalidad. El niño refuerza la conexión entre el hecho que está experimentando ("caerse") y su verbalización ("me caigo") al tiempo que enfatiza su molestia, emitida por la interjección ay, y la permea al asertivo en un proceso de protomodalización.

\footnotetext{
$8 \quad$ En el habla adulta el que como modalizador también aparece en muchas ocasiones como réplica de un enunciado precedente. En nuestro caso, fundamentalmente es réplica de un hecho que está ocurriendo en el contexto situacional inmediato. Los usos como modalizador del que inicial átono en el habla adulta son analizados, entre otros, por Pons (2003), Garrido (1998), entre otros).
} 
Un análisis similar se aplica en el siguiente caso. Vemos que NER recurre a esta misma estructura, en la que la interjección expresa su desaprobación ante el hecho descrito por el asertivo:

Ejemplo 9. Participantes: NER (niña) y MON (adulta). Edad: NER, 3;03.16. Grabación: mil2_04

*NER: no!

\%spa: \$i:an ;

${ }^{*} \mathrm{MON}$ : no quema?

${ }^{*} \mathrm{MON}$ : venga (.) a comer (.) yo voy a comer .

*NER: ay@i (.) que me cae !

\%spa: \$i:ed ; \$i:sp ;

\%exp: se refiere a la comida

*MON: lo ponemos aquí en la mesa de la cocina?

\section{USOS METAPRAGMÁTICOS ESTRATÉGICOS DE LAS INTERJECCIONES EN EDAD TEMPRANA}

En ocasiones los niños movilizan estratégica y teatralmente las funciones pragmáticas anteriormente descritas. Estos usos son prueba de la conciencia metapragmática que empieza a aflorar en edad temprana. Aunque los ejemplos de este tipo encontrados en el corpus son escasos, dada la corta edad de los participantes, consideramos que son dignos de mención y análisis. Su relevancia radica en que los niños pueden usar estratégicamente las interjecciones como marcadores en su discurso para proyectar diferentes fuerzas ilocucionarias hacia eventos teatralizados en el contexto inmediato. Esto es, el niño simula y provoca de forma ficticia una acción con finalidad lúdica y las interjecciones forman parte estratégica de este "peculiar guión". Por ejemplo, en el caso siguiente, el niño moviliza la interjección para proyectar la molestia ante un hecho que él provoca teatralmente: simular que la botella con la que está jugando está a punto de caer:

Ejemplo 10. Participantes: JOR (niño), XUN (niño) y CHE (adulta). Edad: XUN, 2;11.15. Grabación: vit3b_07

${ }^{*} \mathrm{CHE}$ : mira Xulián cómo se llama ésto ?

*XUN: un anillo .

\%spa: \$i:sa ;

${ }^{*}$ CHE: un anillo .

*XUN: uy@i me cae el agua uy@i .

\%spa: \$i:em ; \$i:st ;

\%com: el niño juega con el agua y simula lúdicamente que le va a caer 


\section{Conclusiones}

El análisis de los datos muestra cómo afloran las funciones pragmáticas de las interjecciones en edad temprana y su papel en el desarrollo de la competencia comunicativa y social de los niños (Ochs y Schieffelin 1986). Así, este estudio revela que las interjecciones propias emotivas tienen una especial relevancia cuantitativa y cualitativa en edad temprana.

Los resultados del análisis cuantitativo muestran que estas partículas se usan para expresar cuatro tipos de fuerzas ilocucionarias, a saber: expresar dolor o molestia (EM), expresar emoción positiva (EN), exclamar en desaprobación (ED) y expresar sorpresa (ES). Asimismo, el predominio cuantitativo de las interjecciones como recurso para expresar emociones en el periodo comprendido entre los 1;05 y 2;11 ańos-cuadro 4 - es una prueba del papel clave que desempeñan en el desarrollo pragmático puesto que estos usos son una ilustración importante de los puentes que se tienden entre la fase preidiomática y la fase idiómatica.

La investigación cualitativa demuestra que las interjecciones constituyen actos de habla proto-expresivos cuyo funcionamiento en edad temprana se limita exclusivamente al plano contextual inmediato. Su significado deíctico y su uso contextual local constituirá la base de la primera fase del desarrollo de las intenciones comunicativas en edad temprana (Prego et al. 2011). En este sentido, el análisis pragmático realizado evidencia cómo los niños movilizan las interjecciones como marcadores proyectivos, esto es, con función deíctica para "proyectar" sus emociones, actitudes, acciones o sentimientos hacia eventos situados en el contexto inmediato y compartido por los interlocutores.

Los resultados de este trabajo revelan que, ligada a la función deíctica, afloran tres tipos de funciones pragmáticas: proyectiva contextual, proyectiva proto-textual y proyectiva proto-modalizadora. Estas tres funciones emergen sucesivamente y con diferentes intensidades. Así, la función proyectiva contextual y las formas más básicas de la proyectiva proto-textual (interjección+asertivo) aparecen en nuestros datos en torno a los 1;10 ańos. En cambio, las proyectivas proto-textuales que transcienden el turno para marcar cohesión en el discurso son muy escasas y empiezan a aparecer muy avanzados los 2;0 ańos, cerca de los 3;0. Y, por último, la función de protomodalidad se produce con interjecciones que expresan desaprobación y malestar y no se rastrean en el corpus hasta los 3;0 años.

Así, el hecho de que hasta los 2;0 años las interjecciones actúen como elementos deícticos que se movilizan como constituyentes únicos de turno y que, por otro lado, la función de proto-modalidad o funciones proto-textuales más avanzadas emerjan en torno a los 3;0 años son fases del recorrido del desarrollo pragmático en edad temprana. Este recorrido empieza con el manejo contextual y deíctico de los recursos comunicativos y progresa hacia la posibilidad de gestionar el discurso utilizando las interjecciones como marcadores de cohesión que transcienden el turno (funciones proto-textuales avanzadas) o marcadores de proto-modalización.

En definitiva, los resultados de este trabajo contribuyen a la comprensión del desarrollo pragmático y, por lo tanto, pueden aplicarse a la valoración y evaluación del desarrollo comunicativo en edad temprana. 


\section{Obras CitAdas}

Ameka, Felix. 1992. "Interjections: The universal yet neglected part of speech". Journal of Pragmatics 18.2/3: 101-118.

Alonso-Cortés Fradejas, Ma Dolores. 2006. "Lo lingüístico y lo paralingüístico en los primeros estudios sobre adquisición de lenguaje". En Milka Villayandre Llamazares, Ed., Actas del XXXV Simposio Internacional de la Sociedad Española de Lingüistica. León: Universidad de León. 48-65.

Asano, Yoshiteru. 1997. "Acquisition of English interjections ouch, yuck, and oops in Early Childhood". Colorado Research in Linguistics 15: 1-15.

Barriga Villanueva, Rebeca. 1992. "De las interjecciones, muletillas y repeticiones: su función en el habla infantil". En Rebeca Barriga Villanueva y Josefina García Fajardo, Eds., Reflexiones lingüisticas y literarias, Vol. I, Lingüística. México, DF: El Colegio de México. 99-113.

Blas Arroyo, José Luis. 1995. "La interjección como marcador discursivo: el caso de eh". Anuario de Lingüistica Hispánica 11: 81-117.

Bloom, Lois. 1973. One word at a time: The use of single word utterances before syntax. The Hague, Netherlands: Mouton.

Bloomfield, Leonard. 1933. Language. New York: Henry Holt.

Brandenburg, George. 1915. "The language of a three years old child". Pedagogical Seminary 22: 89-120.

Bühler, Karl. 1934. Sprachtheorie. Jena: Fischer.

Diessel, Holger \& Michael Tomasello. 2001. "The acquisition of finite complement clauses in English: a corpus-based analysis". Cognitive Linguistics 12.2: 97-141.

Díez-Itza, Eliseo, Catherine Snow \& Brian MacWhinney. 1999. "La metodología RETAMHE y el proyecto CHILDES: breviario para la codificación y análisis del lenguaje infantil". Psicothema 11.3: 517-530.

Ehlich, Konrad. 1986. Interjektionen. Niemeyer, Tübingen.

Garrido, Joaquín. 1998. "Discourse structure in grammar". Estudios Ingleses de la Universidad Complutense 6: 49-63.

Givón, Talmy. 2009. The genesis of syntactic complexity: diachrony, ontogeny, neurocognition, evolution. Amsterdam: John Benjamins.

Goffman, Erving. 1981. "Responses cries”. En Forms of talk. Oxford: Blackwell. 78-122.

Gopnik, Alison. 1982. "Words and plans: early language and the development of intelligent action". Journal of Child Language 9.2: 303-318.

Iordanskaja, Lidija \& Igor Mel'čuk. 1995. "Traitement lexicographique de deux connecteurs textuels du français contemporain. En fait vs en réalitể. En Hava Bat-Zeev Shyldkrot y Lucien Kupferman, Eds., Tendances Récentes en Linguistique Française et Générale. Amsterdam: John Benjamins. 211-236.

Jakobson, Roman. 1960. "Linguistics and poetics". En Thomas Sebeok, Ed., Style in language. Cambridge, MA: MIT Press. 350-377. 
Jespersen, Otto. 1924. The philosophy of grammar. London: Allen and Unwin.

MacWhinney, Brian. 2000. The CHILDES Project. Tools for analyzing talk. $3^{\text {rd }}$ edition. Mahwah, N.J.: Lawrence Erlbaum Associates.

MacWhinney, Brian \& Catherine Snow. 1985. "The Child Language Data Exchange System". Journal of Child Language 12.2: 271-296.

Meng, Katharina \& Susanne Schrabback. 1999. "Interjections in adult-child discourse: The cases of German HM and NA". Journal of Pragmatics 31.10: 1263-1287.

Montes, Rosa Graciela. 1999. "The development of discourse markers in Spanish: Interjections". Journal of Pragmatics 31.10: 1289-1319.

Nice, M. 1917. "The speech development of a child from eighteen months to six years". Pedagogical Seminar 24: 204-243.

Ninio, Anat, Catherine Snow, Barbara A. Pan \& Pamela R. Rollins. 1994. "Classifying communicative acts in children's interaction". Journal of Communication Disorders 27: 157-188.

Ninio, Anat \& Polly Wheeler. 1986. "A manual for classifying verbal communicative acts in mother-child interaction". Transcript Analysis 3: 1-83.

Ochs, Elinor \& Bambi Schieffelin. 1986. Language socialization across cultures. Cambridge: Cambridge University Press.

Plunkett, Kim \& Sven Strömqvist. 1992. "The acquisition of Scandinavian languages". En Dan I. Slobin, Ed., The crosslinguistic study of language acquisition, vol. 3. Hillsdale, NJ: Lawrence Erlbaum. 457-556.

Pons Bordería, Salvador. 2003. "Que inicial átono como marca de modalidad". ELUA. Estudios de Lingüistica Universidad de Alicante 17: 531-545.

Prego Vázquez, Gabriela, Montserrat Souto Gómez \& Beatriz Dieste Quiroga. 2011. "El desarrollo pragmático: intenciones y acción comunicativa”. En Milagros Fernández Pérez, Coord., Lingüistica de corpus y adquisición de la lengua. Madrid: Arco Libros. 149-203.

Preyer, William T. 1882. El alma del niño. Madrid: Ediciones Jorro.

Rodríguez Ramalle, Teresa María. 2007. "Las interjecciones llevan complementos, vaya que sí! Análisis de las interjecciones con complemento en el discurso". Español actual: Revista de español vivo 87: 111-125.

Schegloff, Emanuel. 1982. "Discourse as an interactional achievement: Some uses of uh huh and other things that come between sentences". En Deborah Tannen, Ed., Analyzing discourse: Text and talk. Washington, D.C.: Georgetown University Press. 71-93.

Schiffrin, Deborah .1987. Discourse Markers. Cambridge: Cambridge University Press.

Searle, John R. 1969. Speech Acts: an Essay in the Philosophy of Language. Cambridge: Cambridge University Press.

Strange, Ulrike. 2009. The Acquisition of Interjections in Early Childhood. Hamburg: Diplomica Verlag GmbH.

Tesnière, Luciene. 1976. Eléments de syntaxe structurale. París: Klinsieck.

Vázquez Veiga, Nancy. 2003. Marcadores discursivos de recepción. Colección Lucus Lingua, 
ESTUDIOS FILOLÓGICOS

Anexos de Moenia. Revista Lucense de Lingüistica y Literatura, no 13. Santiago de Compostela: Universidade de Santiago de Compostela.

Wilkins, David P. 1992. “Interjections as Deictics”. Journal of Pragmatics 18.2/3: 119-158. Yairi, Ehud. 1981. "Disfluencies of normally two-year-old children". Journal of Speech and Hearing Research 24: 490-495. 